\title{
Experiências de médicos de família e comunidade no cuidado com a saúde de pacientes lésbicas, gays, bissexuais, travestis e transexuais
}

\author{
Experiences of family and community doctors in the health \\ care of lesbian, gay, bisexual and transgender patients
}

\section{Experiencias de médicos de familia y comunitarios en el cuidado de la salud de los pacientes lesbianas, gays, bisexuales y personas transexuales}

\author{
Joao Antonio Smania Gomes ${ }^{1}$ (1), Zeno Carlos Tesser Junior² \\ `Escola de Saúde Pública de Florianópolis - Florianópolis (SC), Brasil. \\ 2Universidade Federal de Santa Catarina - Florianópolis (SC), Brasil.
}

\section{Resumo}

Introdução: A comunidade lésbicas, gays, bissexuais, travestis e transexuais e suas particularidades associadas à saúde foram ignoradas por muitos anos. Embora a homossexualidade e a transexualidade não sejam mais consideradas doenças, ainda prevalece marginalização de muitas pessoas lésbicas, gays, bissexuais, travestis e transexuais a nível sócio-econômicocultural e de acesso aos serviços de saúde. No que tange ao acesso à saúde, o primeiro contato do paciente Lésbicas, Gays, Bissexuais, Travestis e Transexuais dentro do sistema de saúde pode ser através do médico de família e comunidade. Objetivo: Analisar as experiências dos médicos de família e comunidade no atendimento às pessoas lésbicas, gays, bissexuais, travestis e transexuais na atenção básica da rede municipal de saúde em uma cidade no Sul do Brasil. Métodos: Desenvolveram-se dois grupos focais (13 profissionais no total), um deles constituído de seis médicos de família e comunidade autodeclarados heterossexuais e cisgêneros e outro grupo constituído de sete médicos de família e comunidade autodeclarados lésbicas, gays, bissexuais, travestis e transexuais, em julho de 2019. Resultados: Os participantes consideraram importante a temática da saúde lésbicas, gays, bissexuais, travestis e transexuais na atenção primária, embora ela tenha sido pouco explorada nos seus cursos de graduação. Relataram que as principais demandas dos pacientes lésbicas, gays, bissexuais, travestis e transexuais são as de saúde mental, violência e infecções sexualmente transmissíveis. Apontaram que possuem dificuldades em abordar questões que envolvem sexualidade e identidade de gênero em suas consultas. Conclusões: Os resultados reforçam a necessidade de os médicos de família e comunidade conhecerem especificidades da população lésbicas, gays, bissexuais, travestis e transexuais. Sugere-se que a temática da saúde da população lésbicas, gays, bissexuais, travestis e transexuais seja mais ensinada nos cursos de graduação em Medicina.

Palavras-chave: Minorias sexuais e de gênero; Medicina de família e comunidade; Atenção primária à saúde.

Como citar: Gomes JAS, Tesser Junior ZC. Experiências de médicos de família e comunidade no cuidado com a saúde de pacientes LGBT. Rev Bras Med Fam Comunidade. 2022;17(44):2407. https://doi.org/10.5712/ rbmfc17(44)2407

Autor correspondente: Joao Antonio Smania Gomes E-mail: joaosmania@gmail.com Fonte de financiamento: não se aplica. Parecer CEP: CAAE 16080619.4.0000.5360. Procedência: não encomendado. Avaliação por pares: externa.

Recebido em: 21/02/2020.

Aprovado em: 07/11/2021. 


\begin{abstract}
Introduction: The lesbian, gay, bisexual, transvestite and transsexual community and its health-related particularities were ignored for many years. Although homosexuality and transsexuality are no longer considered diseases, the marginalization of many lesbians, gays, bisexuals, transvestites and transsexuals at the socioeconomic-cultural level and with regard to access to health services still prevails. As to access to health care, the first contact of lesbian, gay, bisexual, transvestite and transsexual patients within the health care system can be through the family doctor. Objective: To analyze the experiences of family and community doctors with lesbian, gay, bisexual, and transgender people in the primary care scenario of the public health network in a city in southern Brazil. Methods: In July 2019, two focus groups were arranged (13 professionals in total), one of which consisted of 6 self-declared heterosexual and cisgender family doctors and another group consisting of 7 self-declared lesbian, gay, bisexual and transgender family doctors. Results: Participants considered the issue of lesbian, gay, bisexual, and transgender health in primary care important, but added that it was very little studied in their undergraduate/graduate training. They reported that the main demands of lesbian, gay, bisexual and transgender patients are mental health, violence and sexually transmitted infections. They pointed out that they have difficulties in addressing issues involving sexuality and gender identity during their office visits. Conclusions: The results reinforce the need for family and community doctors to understand better specifics of the lesbian, gay, bisexual, and transgender population. It is suggested that the health issues of the lesbian, gay, bisexual, and transgender population be more taught in undergraduate and graduate medical courses.
\end{abstract}

Keywords: Sexual and gender minorities; Family practice; Primary health care.

\title{
Resumen
}

Introducción: Introducción: La comunidad lesbiana, gays, bisexuales, travestis y transexuales y sus particularidades relacionadas con la salud fueron ignoradas durante muchos años. Si bien la homosexualidad y la transexualidad ya no se consideran enfermedades, aún prevalece la marginación de muchas personas lesbianas, gays, bisexuales, travestis y transexuales en términos socio-económico-culturales y de acceso a los servicios de salud. En cuanto al acceso a la asistencia sanitaria, el primer contacto de las pacientes lesbianas, gays, bisexuales, travestis y transexuales dentro del sistema sanitario puede ser a través del médico de familia y comunidad. Objetivo: Analizar las experiencias de los Médicos de Familia y Comunidad en el cuidado de las personas lesbianas, gays, bisexuales y personas transexuales en la atención primaria de la red de salud municipal en una ciudad del sur de Brasil. Métodos: Se desarrollaron dos grupos focales (13 profesionales en total), uno de los cuales consistió en 6 Médicos de Familia y Comunidad autodeclarados heterosexuales y cisgénero y otro grupo compuesto por 7 Médicos de Familia y Comunidad lesbianas, gays, bisexuales y personas transexuales autodeclarados en julio de 2019. Resultados: Los participantes consideraron que el tema de la salud lesbianas, gays, bisexuales y personas transexuales era importante en la atención primaria, aunque fue poco explorado en sus cursos de formación. Informaron que las principales demandas de las pacientes lesbianas, gays, bisexuales y personas transexuales son la salud mental, la violencia y las infecciones de transmisión sexual. Señalaron que en sus consultas tienen dificultades para abordar cuestiones relacionadas con la sexualidad y la identidad de género. Conclusiones: Los resultados refuerzan la necesidad de que los Médicos de Familia y Comunidad conozcan las especificidades de la comunidad lesbianas, gays, bisexuales y personas transexuales. Se sugiere que el tema de salud lesbianas, gays, bisexuales y personas transexuales sea más enseñado en la formación médica.

Palabras clave: Minorías sexuales y de género; Medicina familiar y comunitaria; Atención primaria de salud.

\section{INTRODUÇÃO}

A comunidade de lésbicas, gays, bissexuais, travestis e transexuais (LGBT) e suas particularidades associadas à saúde foram ignoradas por muitos anos. ${ }^{1} \mathrm{O}$ simples fato de se identificar como um homem gay ou uma mulher lésbica era considerado doença até o início dos anos 1970 pela Associação Americana de Psiquiatria $(A A P)^{2}$ e até o início dos anos 1990 pela Organização Mundial da Saúde (OMS). ${ }^{3}$ Ademais, as pessoas trans eram classificadas pela OMS como portadoras de transtorno de identidade de gênero até o ano de 2018. Foi então que tal característica deixou de ser classificada como parte do escopo de doenças mentais e passou a ser tratada como uma "condição relacionada à saúde sexual" na atualização do Código Internacional de Doenças (CID-11). ${ }^{4}$

Embora a homossexualidade e a transexualidade não sejam mais consideradas doenças pelas ciências da saúde, ainda prevalece uma cultura de desinformação sobre o tema, construída ao longo dos anos e que gera preconceitos e determina a marginalização de muitas pessoas LGBT em nível socioeconômico-cultural e no acesso aos serviços de saúde. ${ }^{5}$ 
Como importantes determinantes sociais de saúde, é imprescindível diferenciar orientação sexual e identidade de gênero. ${ }^{5} \mathrm{~A}$ orientação sexual "(..) é a capacidade de ter, sentir ou desenvolver atração e/ ou relação emocional, afetiva ou sexual por outras pessoas", ${ }^{6}$ podendo ser classificada (em um sistema binário de gênero - masculino e feminino) em homossexual (pessoas do mesmo gênero atraem-se), heterossexual (gêneros opostos atraem-se) e bissexual (existe atração por ambos os gêneros).

Já a identidade de gênero "(...) é a expressão de uma identidade construída a partir de como a pessoa se reconhece e/ou se apresenta, que pode corresponder ou não ao seu corpo biológico", 6 independentemente de modificações corporais, que podem existir ou não. Existem inúmeras identidades descritas, entre elas homens e mulheres trans, travesti, queer, pessoas não binárias etc. Ressalta-se que não existe associação direta entre a orientação sexual e a identidade de gênero; por exemplo, pessoas trans podem ser hetero, homo ou bissexuais. ${ }^{5}$

Referimo-nos às pessoas trans neste artigo como aquelas que se identificam com o gênero diferente do determinado ao nascimento; por exemplo, a mulher trans, ao nascimento, foi determinada como homem pela genitália tipicamente masculina, porém identifica-se como mulher, de modo que é uma mulher trans. Outro exemplo: homem trans, ao nascimento determinado como mulher pela genitália tipicamente feminina, porém identifica-se como homem, portanto é um homem trans. Os cisgêneros (o termo "cis" vem do latim e significa "do mesmo lado que") são as pessoas que reconhecem a própria identidade de gênero de acordo com o sexo biológico. Por exemplo, homens designados assim por genitália tipicamente masculina ao nascer e que se identificam com o gênero masculino; ou mulheres designadas assim pela genitália de nascimento e que se identificam com o gênero feminino. ${ }^{7}$ Também ressaltamos que a heteronormatividade será referida como um sistema no qual as relações românticas e de condutas sexuais são constituídas entre homens e mulheres cisgêneros, de forma heterossexual, e que é entendido como a ordem "natural" culturalmente aceita. ${ }^{8}$

A identidade de gênero e a orientação sexual foram incluídas em análise como determinantes sociais da saúde da população LGBT na 13ª Conferência Nacional de Saúde, em 2007. Dessa conferência resultou uma série de recomendações aos profissionais de saúde com relação ao cuidado com a população LGBT, sendo aprovada, nos anos subsequentes, a Política Nacional Integral de Saúde LGBT (PNSILGBT), que aprofunda ainda mais o tema e as diretrizes de cuidado dessa população. ${ }^{9}$

Em relação ao acesso à saúde, o primeiro contato médico do paciente LGBT no sistema de saúde pode ser por meio de uma das especialidades que mais lida rotineiramente com as minorias sociais em saúde: o médico de família e comunidade (MFC). Entre os atributos associados a essa especialidade da Medicina, tem-se que o MFC deve promover acesso amplo e ilimitado aos usuários, lidando com todos os tipos de problemas de saúde independentemente de idade, gênero ou qualquer outra característica do paciente.

Como detentor do título de tal especialidade, o MFC deve ainda, por definição, promover o empoderamento do paciente, desenvolver a continuidade do cuidado de forma longitudinal, bem como lidar com problemas de saúde em suas dimensões físicas, psicológicas, sociais, culturais e existenciais. ${ }^{10}$

Nesse sentido, considerando-se as inúmeras disparidades de saúde vividas pela população LGBT, ${ }^{11}$ o presente estudo tem por objetivo analisar as experiências dos MFC com relação ao atendimento médico realizado na atenção básica, ou seja, nas Unidades Básicas de Saúde (UBS) da rede municipal, com as pessoas LGBT.

Para isso contaremos com experiências de MFC que são autodeclarados como pertencentes ao grupo LGBT e com MFC que são autodeclarados heterossexuais e cisgêneros, comparando-as de forma a observar as questões/experiências com a população LGBT no cotidiano. Também foram objetivos desta 
pesquisa estabelecer que contato os participantes tiveram com a temática LGBT na educação médica formal e conhecer os principais desafios e avanços que os MFC destacam no atendimento dessa população.

Percebe-se que a literatura científica enfocou mais as experiências de preconceito e estigmatização que as pessoas LGBT sofrem nos serviços de saúde e menos as experiências que os profissionais médicos têm no atendimento a essas pessoas. Sendo assim, conhecer as experiências de MFC no cuidado de pacientes LGBT contribui para a possibilidade de se pensarem diferentes ângulos e perspectivas sobre a saúde da população LGBT. Afinal, os MFC também são interessados em dar visibilidade às demandas e situações de saúde que ocorrem no dia a dia de suas atividades profissionais.

\section{MÉTODOS}

Estudo qualitativo feito com dois grupos focais. Para Minayo, ${ }^{12}$ o grupo focal constitui-se “(...) num tipo de entrevista ou conversa em grupos pequenos e homogêneos", pode complementar a observação participante e as entrevistas semiestruturadas e é útil para a formação de opinião coletiva e a formação de consensos. Para Barbour, ${ }^{13}$ mais do que uma entrevista em grupo, é uma forma de analisar a interação entre os participantes, que devem ser de no mínimo seis por grupo.

Os sujeitos da pesquisa foram 13 MFC atuantes na rede municipal de saúde de uma cidade na região Sul do Brasil. Um grupo foi composto de sete MFC que se autoidentificaram como LGBT, e o outro de seis MFC autoidentificados como heterossexuais cisgêneros. Nenhum participante se identificou como transgênero. As características dos participantes, identificados por códigos para a preservação do sigilo de suas identidades, estão descritas na Tabela 1.

Tabela 1. Caracterização dos médicos de família e comunidade participantes do grupo focal.

\begin{tabular}{ccccccc}
\hline $\begin{array}{c}\text { Identificação do } \\
\text { Participante }\end{array}$ & Grupo & Idade & $\begin{array}{c}\text { Identidade } \\
\text { de Gênero }\end{array}$ & $\begin{array}{c}\text { Orientação } \\
\text { Sexual }\end{array}$ & Estado Civil & $\begin{array}{c}\text { Tempo como } \\
\text { MFC }\end{array}$ \\
\hline P1 & MFC LGBT & 29 & Mulher & Lésbica & Solteira & 2 \\
P2 & MFC LGBT & 52 & Mulher & Lésbica & Casada & 20 \\
P3 & MFC LGBT & 32 & Mulher & Bissexual & Casada & 6 \\
P4 & MFC LGBT & 32 & Mulher & Bissexual & Solteira & 2 \\
P5 & MFC LGBT & 40 & Homem & Gay & Solteiro & 12 \\
P6 & MFC LGBT & 35 & Homem & Gay & Casado & 6 \\
P7 & MFC LGBT & 28 & Homem & Gay & Solteiro & 2 \\
P8 & MFC N-LGBT & 45 & Mulher & Heterossexual & Divorciada & 18 \\
P9 & MFC N-LGBT & 32 & Mulher & Heterossexual & Casada & 4 \\
P10 & MFC N-LGBT & 32 & Mulher & Heterossexual & Casada & 5 \\
P11 & MFC N-LGBT & 43 & Homem & Heterossexual & Casado & 10 \\
P12 & MFC N-LGBT & 44 & Homem & Heterossexual & Casado & 9 \\
P13 & MFC N-LGBT & 42 & Homem & Heterossexual & Casado & 10 \\
\hline
\end{tabular}

MFC: médico de família e comunidade; LGBT: lésbicas, gays, bissexuais, travestis e transexuais.

Para a constituição dos grupos focais, foram contatados os responsáveis pelos MFC do município que, gentilmente, divulgaram aos outros MFC integrantes da rede municipal o convite para participar da pesquisa. Os critérios de inclusão foram: ser médico com titulação em Medicina de Família e Comunidade 
atuando na rede de atenção básica, ter voluntariamente se autodeclarado como LGBT ou heterossexual e não apresentar conflitos de interesse com a temática do estudo.

Os MFC que mostraram interesse em participar e que se autodeclararam como LGBT foram colocados no grupo "MFC LGBT", enquanto os que se autodeclararam heterossexuais foram colocados no grupo "MFC N-LGBT". Os participantes foram separados dessa forma para dar voz a ambos os grupos entre seus pares, de modo que não houvesse risco de supressão de opiniões ou falas por qualquer uma das partes. Dessa forma, ficou privilegiada a homogeneidade dos grupos, que, segundo Westphal, ${ }^{14}$ resulta no aprofundamento das discussões na medida em que os participantes falam de seu lugar específico na sociedade.

$\mathrm{Na}$ parte inicial deste estudo, foi feito o planejamento do roteiro semiestruturado usado nos grupos focais. Esse roteiro, idêntico para os dois grupos, foi composto de cinco guias de tópicos, mostrados a seguir:

1. Como as questões LGBT aparecem no seu cotidiano como MFC na atenção primária?

2. Quando e como você aborda a orientação sexual e a identidade de gênero dos pacientes nas consultas?

3. Quais os desafios que você percebe com relação à temática LGBT como MFC na atenção primária? 4. Quais os avanços que você percebe com relação à temática LGBT como MFC na atenção primária?

5. Você possui sugestões para melhorar o atendimento à população LGBT na atenção primária?

Cada grupo participou de um encontro para a realização dos grupos focais, que ocorreram em datas separadas, no período noturno, no mês de julho de 2019. O local dos dois encontros, um para cada grupo, foi uma UBS da cidade onde a pesquisa foi realizada. A duração de cada encontro foi de aproximadamente $90 \mathrm{~min}$. Antes de iniciarem os grupos focais, os participantes preencheram questões que objetivaram traçar os seus perfis sociodemográficos.

Os grupos focais foram feitos por dois pesquisadores. Um deles ficou responsável pela moderação; assim, quando necessário, incentivou a participação de todos, limitou o predomínio de algum participante e restringiu a conversa ao escopo da pesquisa. Enquanto isso, o outro pesquisador, sem interação com o grupo, teve a função de relator, anotando as opiniões, experiências, observações e outras questões levantadas pelos participantes. Destaca-se que ambos pesquisadores não fizeram quaisquer tipos de julgamentos durante as reuniões.

As falas dos participantes foram capturadas em áudio utilizando-se um gravador digital. No dia seguinte, todas elas foram transcritas na íntegra pelo relator dos grupos. Após a realização dos grupos focais, os pesquisadores reuniram-se para uma discussão sobre os dados levantados.

Para a análise dos dados foi utilizada a análise temática, conforme sugerida por Minayo, ${ }^{12}$ realizada em três etapas. Na primeira etapa, a pré-análise, após leitura exaustiva do material coletado foram selecionadas palavras-chaves e frases importantes que seriam usadas como unidades de contexto, com base nas quais seriam feitos os recortes, a categorização e a codificação dos dados, bem como a seleção dos conceitos teóricos.

$\mathrm{Na}$ segunda etapa, a de exploração, foi feita a categorização do material, que teve seu texto reduzido a falas ou expressões consideradas significativas, as quais foram classificadas e agrupadas em oito categorias temáticas, mostradas nos resultados. Na terceira etapa, a de tratamento e interpretação de dados, foram trabalhados os significados dos resultados brutos, dos quais foram feitas inferências e interpretações. Destaca-se que se optou pela variante da técnica que não submete os dados a operações estatísticas, como sugerido por Minayo. ${ }^{12}$ 
O presente estudo foi aprovado pelo Comitê de Ética em Pesquisa com Seres Humanos pertinente segundo o número de Certificado de Apresentação para Apreciação Ética (CAAE) 16080619.4.0000.5360 e pela Comissão de Acompanhamento dos Projetos de Pesquisa em Saúde da Secretaria Municipal da cidade onde ocorreu o estudo. Todos os participantes assinaram o Termo de Consentimento Livre e Esclarecido.

\section{RESULTADOS E DISCUSSÃO}

Com base na análise temática, emergiram das falas dos participantes as seguintes categorias temáticas:

1. importância da temática de saúde LGBT na atenção primária;

2. saúde mental das pessoas LGBT;

3. violência doméstica contra a população LGBT;

4. infecções sexualmente transmissíveis (IST);

5. dificuldade dos MFC em abordar questões que envolvem sexualidade e identidade de gênero;

6. ensino da temática LGBT e currículo oculto nos cursos de Medicina;

7. avanços e desafios na atenção à saúde da população LGBT; e

8. sugestões para melhoria de atendimento às pessoas LGBT.

Destacamos que são comuns limitações de ordem metodológica nas pesquisas realizadas sobre a temática LGBT em todo o mundo, seja pela falta de interesse em participar nas pesquisas, seja pelo receio que as pessoas possuem de serem vítimas de preconceito ao participarem delas. Outra limitação é que os MFC se autosselecionaram para este estudo por meio de um convite, portanto nossa amostra pode não representar a experiência de todos os MFC, mas daqueles que já estão mais familiarizados e atentos às demandas em saúde das pessoas LGBT.

\section{Importância da temática lésbicas, gays, bissexuais, travestis e transexuais na atenção primária}

Todos os participantes, dos dois grupos, consideraram importante a temática de saúde LGBT. No grupo de heterossexuais cisgêneros, ressaltou-se que na cidade da pesquisa há um ambulatório que atende à população transgênera/travesti na Atenção Primária. O ambulatório citado foi instituído, de forma voluntária, por residentes de MFC que vivenciaram prática similar em estágio no Uruguai, tendo sido assumido e ampliado por outros MFC. ${ }^{14}$

\section{Saúde mental das pessoas lésbicas, gays, bissexuais, travestis e transexuais}

Com relação às principais formas em que observam as demandas relacionadas à orientação sexual e à identidade de gênero, os dois grupos relataram a associação com queixas relacionadas à saúde mental dos pacientes, seja como sofrimento por não aceitação na família, seja por dificuldades na própria aceitação e pelo abuso de substâncias.

Tais dados foram ao encontro da literatura mundial, a qual mostra que lésbicas, bissexuais e gays apresentam 1,5 vezes mais chance de desenvolver ansiedade e depressão que heterossexuais, sendo a 
chance de tentativas de suicídio quatro vezes maior em homens gays e bissexuais quando comparados aos heterossexuais. ${ }^{15} \mathrm{O}$ risco de suicídio na população transgênera é ainda maior. Uma pesquisa nacional constatou que $41 \%$ dos entrevistados transgêneros já haviam tentado suicídio, com taxas maiores ainda em indivíduos mais jovens. ${ }^{16}$

Outra forma de sofrimento mental pode aparecer no abuso de substâncias, que também é mais comum entre pessoas LGBT: mulheres lésbicas e bissexuais apresentam taxas mais altas de uso de tabaco e exposição passiva ao tabaco, além de álcool e dependência química. ${ }^{17} \mathrm{Um}$ estudo estadunidense mostrou que, comparados a heterossexuais, as chances de abuso de substância durante a vida eram três vezes maiores em mulheres lésbicas e 1,6 vezes maior em homens gays. ${ }^{18} \mathrm{Na}$ população trans, os dados de abuso são ainda maiores e as substâncias químicas usadas ainda mais variadas e perigosas. ${ }^{16}$

\section{Violência contra a população lésbicas, gays, bissexuais, travestis e transexuais}

A questão da violência contra as pessoas LGBT também apareceu como uma demanda das pessoas LGBT na atenção primária, segundo os MFC participantes da pesquisa. Vale destacar que a violência doméstica - física, psicológica ou sexual - é tão prevalente quanto em relacionamentos heterossexuais, havendo estudos estadunidenses que demonstraram porcentagem significativa de casais de mesmo gênero que reportaram esse tipo de violência. ${ }^{19,20}$ Entretanto, com relação à violência externa, o projeto Trans Murder Monitoring. ${ }^{21}$ ("Monitoramento de Assassinatos Trans") documentou mais de 2 mil assassinatos em 66 países entre 2008 e 2016, o equivalente a um assassinato a cada dois dias.

No Brasil, o Grupo Gay da Bahia (GGB) ${ }^{22}$ produz relatórios anuais sobre pessoas LGBT mortas por terem sido vítimas da homofobia. Nos dados por eles divulgados, percebe-se, desde os anos 2000, um aumento nessa contabilização. Em 2018, foram 420 mortes, com predominância de gays, seguidos por pessoas trans/travestis e lésbicas. Pelo método de coleta dos dados do GGB, que se dá por notícias sobre a violência homofóbica, entende-se, portanto, que há subestimação desses dados.

Ao analisar os dados disponíveis pelo Ministério da Saúde (MS), disponíveis no TabNet Win32 3.0, observa-se que, entre os anos de 2012 e 2016, o crescimento do número de homicídios contra LGBT aumentou no Brasil. Quando comparado com a população em geral, no mesmo período, o número de homicídios contra pessoas LGBT foi 13 vezes mais alto. Esses crimes são um problema de saúde pública que vitimiza na maioria das vezes jovens, principalmente transgênerosm. ${ }^{23}$

\section{Infecções sexualmente transmissíveis}

Outro recorte importante ressaltado pelos dois grupos de participantes foi o alto número de pacientes gays portadores do vírus da imunodeficiência humana (HIV). Um participante do grupo MFC LGBT relata que, no território adscrito de sua equipe, a maioria dos pacientes homens homossexuais de sexualidade aberta para a comunidade frequentam o Centro de Saúde para tratamento da infecção crônica pelo vírus do HIV. Com relação às infecções de transmissão sexual do vírus HIV, no Brasil, houve crescimento na detecção entre os anos de 2007 e 2017, predominando entre o grupo masculino em relações homossexuais e bissexuais, com aumento na detecção da faixa etária de 13-39 anos. Já a infecção feminina se concentrou em relações heterossexuais (>95\%). ${ }^{24}$ Importante ressaltar que, muitas vezes, os homens cisgênero homossexuais são protagonistas de estudos científicos apenas com esse enfoque. Uma revisão dos projetos de pesquisa financiados pelo National Institute of Health (NIH) estadunidense 
em 2014, que avaliou 628 estudos entre 1998 e 2011 relacionados à saúde LGBT, mostrou que quase $80 \%$ dessas pesquisas eram focadas em HIVIAIDS. O estudo concluiu que a falta de pesquisas relacionadas à saúde LGBT pelo NIH contribui para a perpetuação de inequidades em saúde. ${ }^{25}$

\section{Dificuldade dos MFC em abordar questões que envolvem sexualidade e identidade de gênero.}

Quando Ihes foi perguntado como realizam a abordagem de orientação sexual e identidade de gênero, ambos os grupos divergiram entre si nas respostas e experiências. Nos dois grupos, membros relataram que não têm o hábito de perguntar rotineiramente a seus pacientes sobre sua sexualidade ou identidade de gênero, enquanto outros relataram perguntar a todos os seus pacientes em consultas pertinentes, como as tais "consultas de rotina". Um participante do grupo LGBT discorreu sobre sua dificuldade:

"Eu ia falar dessa questão da heteronormatividade, acho que mesmo sendo um homem gay, muitas vezes eu acho que patino um pouco na abordagem. A gente até discutiu com os residentes recentemente sobre a hora da consulta mesmo, como abordar isso de uma maneira que fique tranquila, porque às vezes na correria fica corrido" (P6).

O grupo focal LGBT concordou com essa posição e participantes acrescentaram que acreditam que o poder de empatia dos médicos LGBT é maior por também sentirem na pele o preconceito, porém eles têm dificuldades por não serem treinados. Um dos participantes relatou que sente que está invadindo a privacidade do paciente. Outro relatou:

"É uma coisa projetada ao longo do tempo, hoje ciente disso, o movimento que eu faço é tentar desconstruir isso e abordar de maneira mais natural possível 'você tem uma pessoa?', 'você transa com homens? E com mulheres?'. É natural" (P3).

Participantes de ambos os grupos relataram experiências com mulheres lésbicas e bissexuais nas quais presumiram a heteronormatividade e ofereceram métodos contraceptivos para essas pacientes. Dados brasileiros mostram ainda a dificuldade no acesso das mulheres lésbicas e bissexuais aos serviços de saúde. Cerca de $40 \%$ delas não revelaram sua orientação sexual. Entre as que revelaram, 28\% relataram maior rapidez na duração do atendimento médico. ${ }^{26}$

\section{Ensino da temática lésbicas, gays, bissexuais, travestis e transexuais e currículo ocul- to nos cursos de Medicina}

Os participantes dos dois grupos foram unânimes em reconhecer a falta de conteúdo sobre a saúde de pessoas LGBT nos espaços de ensino formal da Medicina, tanto na graduação quanto em cursos de especialização. Contudo, apenas no grupo MFC LGBT foram apontadas questões da temática LGBT como integrantes de um currículo oculto que se deu após manifestações homofóbicas por parte dos professores.

Entende-se por currículo oculto as experiências de aprendizado sobre normas, valores e tendências que se dão nas rotinas e nas relações sociais que circundam os espaços institucionais de ensino, sem 
estarem manifestos nos currículos formais dos cursos. ${ }^{27}$ Um exemplo foi a expressão "esse é fruta", dita por um dos professores dos participantes quando saiu um paciente gay do consultório.

Os participantes do grupo MFC LGBT ressaltam também que as discussões sobre as especialidades que fariam após se formarem eram carregadas de preconceitos. Quando, por exemplo, um estudante homem declarava interesse em Pediatria, ouvia frases do tipo: "Não é homem, porque escolheu uma especialidade que é para mulheres" (P5).

Outro participante cita o ensinamento da abordagem ao paciente homossexual como grupo de risco: "(...) quando o paciente se identificava como homossexual deveríamos abordar IST e sorologia, agora se era heterossexual e casado, não precisava" (P7).

Tais atitudes percebidas e ressaltadas pelo grupo MFC LGBT durante sua graduação perpetuam preconceitos que, por sua vez, contribuem para um cuidar em saúde de qualidade inferior para essas minorias, além de para a manutenção de conceitos errados para o ensino de futuras gerações de trabalhadores de saúde. ${ }^{28}$

Nesse sentido, é importante atentar para o fato de que os alunos de Medicina possuem altas taxas de preconceito contra pessoas LGBT. Em 2017, um estudo com 391 graduandos do primeiro ao oitavo semestre de Medicina em uma universidade pública da região Sul do Brasil mostrou, por exemplo, que $74,9 \%$ dos estudantes concordam que o sexo entre dois homens é errado e que $83,9 \%$ afirmam que as meninas masculinas deveriam receber tratamento. ${ }^{29}$

\section{Avanços e desafios na atenção à saúde da população lésbicas, gays, bissexuais, tra- vestis e transexuais}

Nos dois grupos, os participantes citam a dificuldade de acesso aos serviços de saúde como o principal desafio na atenção a saúde das pessoas LGBT. Ressaltam que o preconceito pode começar na recepção, antes mesmo de a pessoa chegar aos médicos, quando, por exemplo, a identidade de gênero e o nome social de uma pessoa trans não são respeitados pelos seguranças e recepcionistas.

Uma pesquisa com diferentes trabalhadores da saúde como dentistas, enfermeiros e agentes comunitários de uma rede municipal de saúde mostrou que eles possuem representações sociais das pessoas LGBT estabelecidas em morais religiosas e heteronormativas, relacionando-as com estereótipos, IST e promiscuidade..$^{30}$

Os grupos ampliam a noção de dificuldade de acesso para o preconceito presente na sociedade. Um participante do grupo MFC N-LGBT relata:

"(...) eu me considero uma pessoa um pouco mais esclarecida e isso talvez me faça difícil de reconhecer, mas ainda assim eu tenho preconceito, eu acho que a gente está sempre olhando, "por que tu fez isso, por que tu agiu assim, e não agiu como habitualmente tu age?", acho que temos que ter esse cuidado, o preconceito da equipe, o preconceito da sociedade, isso traz muita dificuldade, há relatos de muitas pessoas que não procuraram o posto do bairro porque têm vergonha de estar no meio das pessoas próximas, mas por conta das experiências anteriores" (P11).

Os apontamentos realizados pelos profissionais estão em concordância com diversas outras experiências mundiais que indicam que pacientes LGBT vivenciam disparidades no atendimento à saúde quando comparados com a população em geral, ${ }^{31}$ além de apresentarem menos confiança nos serviços de saúde. ${ }^{32}$ 
Já quando os participantes foram indagados sobre os avanços que perceberam com relação ao tema de saúde da população LGBT, ambos os grupos concordam que há avanços da sociedade em geral, maior discussão do tema principalmente entre adultos jovens, maior exposição midiática dedicada ao assunto (como personagens em novelas). Mais especificamente na cidade do estudo, um participante do grupo MFC N-LGBT ressalta que com o ambulatório trans houve uma capacitação para os profissionais que se envolveram. Tais profissionais divulgam mais a temática LGBT pela rede de saúde. Outro participante sublinha que ter o espaço para colocar o nome social no prontuário eletrônico já constitui um avanço.

\section{Sugestões para melhoria de atendimento às pessoas LGBT}

Com relação às sugestões que os profissionais participantes teriam para melhorias com relação à temática LGBT, os grupos foram unânimes em reforçar a importância de institucionalizar e formalizar o ensino sobre saúde LGBT na graduação médica, bem como oferecer capacitações para todos os profissionais de saúde, seja na rede municipal de serviços de saúde, seja em cursos online. Ressaltaram a importância da valorização do profissional que tem esses conhecimentos e da cobrança de que ele tenha uma postura ética perante o paciente LGBT, como ressalta um participante do grupo MFC N-LGBT:

"Acho que, para além de oferecer capacitação, cobrar que a pessoa se atualize com aquilo, mas cobre que a pessoa tenha aquela postura, não adianta oferecer somente o certificado (...) que a avaliação probatória funcionasse, inclusive em contemplar essa questão de ética e profissionalismo, isso na verdade é até profissionalismo, como você é profissionalmente, independente do que for na vida" (P9).

As sugestões apresentadas dialogam diretamente com outros estudos sobre o tema encontrados na literatura mundial, que sugerem também como recursos para a educação: a inclusão de pacientes LGBT em casos simulados a serem estudados como parte da variedade populacional, a melhora dos estudos de sexualidade nos currículos de residência e graduação médica, grupos de apoio patrocinado pelas instituições de ensino, diretrizes institucionais que condenem a discriminação amplamente divulgadas pelas instituições de saúde e medidas a serem tomadas contra indivíduos que pratiquem LGBTfobia nesses ambientes. ${ }^{33}$

\section{CONSIDERAÇÕES FINAIS}

Aimportância do reconhecimento da orientação sexual e da identidade de gênero como determinantes sociais de saúde explica algumas disparidades associadas à saúde das pessoas LGBT. As diferentes formas de discriminação, entre elas a lesbofobia, a gayfobia, a bifobia, a travestifobia e a transfobia, impactam profundamente de forma negativa a saúde de quem as sofre, criando dificuldades que vão do acesso ao sistema de saúde até a manutenção do cuidado de si. ${ }^{5}$

Os grupos focais com participantes MFC heterossexuais cisgêneros e MFC LGBT apresentaram ambos dificuldades com a temática LGBT, que envolveram desde a forma como abordar a saúde LGBT até como prover acesso de qualidade a essa população, o que indica a falibilidade da abordagem da temática nas instituições de ensino em saúde e o preconceito da sociedade, de modo geral, sobre o tema. 
Como perspectivas futuras, mostram-se extremamente necessários mais estudos sobre a saúde das pessoas LGBT para aumentar a visibilidade do tema, no intuito de diminuir iniquidades em saúde para essa população, além de fomentar a discussão sobre o assunto em busca da inserção de seus estudos de modo sistematizado na educação em saúde.

\title{
CONTRIBUIÇÃO DOS AUTORES
}

JASG: Conceituação, Curadoria de Dados, Análise Formal, Escrita - Primeira Redação, Escrita Revisão e Edição. ZCTJ: Conceituação, Curadoria de Dados, Análise Formal, Escrita - Revisão e Edição.

\section{CONFLITO DE INTERESSES}

\author{
Nada a declarar.
}

\section{REFERÊNCIAS}

1. United Nations. Born free and equal: sexual orientation and gender identity in international human rights law. United Nations: New York and Geneva, 2012.

2. Drescher J. Out of DSM: depathologizing homosexuality. Behav Sci 2015;5(4):565-75. https://doi.org/10.3390/bs5040565

3. World Health Organization. The ICD-10 classification of mental and behavioural disorders: diagnostic criteria for research. Genebra: World Health Organization; 1993.

4. The Lancet. ICD-11: a brave attempt at classifying a new world. Lancet 2018;391(10139):2476. https://doi.org/10.1016/ S0140-6736(18)31370-9

5. Brasil. Política Nacional de Saúde Integral de Lésbicas, Gays, Bissexuais, Travestis e Transexuais. Brasília: Ministério da Saúde; 2013.

6. Brasil. Atenção Integral à Saúde da População Trans. Brasília: Ministério da Saúde; 2014.

7. Aultman B. Cisgender. TSQ 2014;1(1-2):61-2. https://doi.org/10.1215/23289252-2399614

8. Wieringa SE. Symbolic subversion. TSQ 2014;1(1-2):210-2. https://doi.org/10.1215/23289252-2400046

9. Brasil. Relatório Final da 13a Conferência Nacional de Saúde. Brasília: Conselho Nacional de Saúde; 1998.

10. Allen J, Gay B, Crebolder H, Heyrman J, Svab I, Ram P. The European definition of general practice/family medicine. Ljubljan: EURACT; 2005.

11. Tesser-Junior ZC, Kovaleski DF. A invisibilidade das pessoas LGBT na atenção a saúde. In: Figueiredo GLA, Martins CHG, Akerman M, org. Vulnerabilidades e Saúde: grupos em cena por visibilidade no espaço urbano. São Paulo: HUCITEC; 2018. p. 123-31.

12. Minayo MCS. O desafio do conhecimento: pesquisa qualitativa em saúde. 12a ed. São Paulo: HUCITEC; 2010.269 p.

13. Barbour R. Doing focus groups. 2007. https://doi.org/10.4135/9781849208956

14. Westphal MF. Uso de métodos qualitativos no estudo de movimentos sociais por saúde. In: Spínola AWP, Sá ENC, Westphal MF, Adorno RCF, Zioni F, org. Pesquisa social em saúde. 1ª ed. São Paulo: Cortez Editora; 1992.

15. King M, Semlyen J, Tai SS, Killaspy H, Osborn D, Popelyuk D, et al. A systematic review of mental disorder, suicide, and deliberate self harm in lesbian, gay and bisexual people. BMC Psychiatry 2008;8:70. https://doi.org/10.1186/1471-244X-8-70

16. Grant JM, Mottet LA, Tanis J, Herman JL, Harrison J, Keisling M. National transgender discrimination survey report on health and health care. National Transgender Discrimination Survey Report on Health and Health Care; 2010.

17. Cochran SD, Bandiera FC, Mays VM. Sexual orientation-related differences in tobacco use and secondhand smoke exposure among US adults aged 20 to 59 years: 2003-2010 National Health and Nutrition Examination Surveys. Am J Public Health 2013;103(10):1837-44. https://doi.org/10.2105/AJPH.2013.301423

18. McCabe SE, West BT, Hughes TL, Boyd CJ. Sexual orientation and substance abuse treatment utilization in the United States: results from a national survey. J Subst Abuse Treat 2013;44(1):4-12. https://doi.org/10.1016/j.jsat.2012.01.007

19. Tjaden P, Thoennes N. Extent, nature, and consequences of intimate partner violence. Atlanta: Centers for disease control and prevention; 2000.

20. Stephenson R, Khosropour C, Sullivan P. Reporting of intimate partner violence among men who have sex with men in an online survey. West J Emerg Med. 2010;11(3):242-6. PMID: 20882143

21. Transrespect versus Transphobia Worldwide [internet]. 2018 [acessado em 11 jan. 2020]. Disponível em: https://transrespect. org/en/trans-murder-monitoring/

22. Michels E, Mott L. Mortes violentas de LGBT no Brasil: relatório 2017. Salvador: Grupo Gay da Bahia; 2018. 
23. Mendes WG, Silva CMFP. Homicídios da população de lésbicas, gays, bissexuais, travestis, transexuais ou transgêneros (LGBT) no Brasil: uma análise espacial. Cien Saude Colet 2020;25(5):1709-22. https://doi.org/10.1590/141381232020255.33672019

24. Brasil. Boletim Epidemiologico HIV Aids 2017. Brasília: Ministério da Saúde; 2018.

25. Deschamps D, Singer B, Boylan JF. LGBTQ Stats: Lesbian, Gay, Bisexual, Transgender, and Queer People by the Numbers. New York: The New Press; 2017. 340 p.

26. Facchini R, Barbosa RM. Dossiê saúde das mulheres lésbicas: promoção da equidade e da integralidade. Belo Horizonte: Rede Feminista de Saúde; 2006.

27. Apple MW. Ideologia e currículo. [Internet]. $3^{a}$ ed. Porto Alegre: Artmed; 2006.

28. Fallin-Bennett K. Implicit bias against sexual minorities in medicine: cycles of professional influence and the role of the hidden curriculum. Acad Med 2015;90(5):549-52. https://doi.org/10.1097/ACM.0000000000000662

29. Moretti-Pires RO, Guadagnin LI, Tesser-Júnior ZC, Campos DA, Turatti BO. Preconceito contra diversidade sexual e de gênero entre estudantes de medicina de $1^{\circ}$ ao $8^{\circ}$ semestre de um curso da região sul do Brasil. Rev Bras Educ Med 2019;43(Suppl 1):557-67. https://doi.org/10.1590/1981-5271v43suplemento1-20190076

30. Silva ALR, Finkle M, Moretti-Pires RO. Representações sociais de trabalhadores da atenção básica à saúde sobre pessoas LGBT. Trab Educ e Saúde 2019;17(2). https://doi.org/10.1590/1981-7746-sol00197

31. Makadon HJ. Ending LGBT invisibility in health care: the first step in ensuring equitable care. Cleve Clin J Med 2011;78(4):2204. https://doi.org/10.3949/ccjm.78gr.10006

32. Fadus M. Mental Health Disparities and Medical Student Education: Teaching in Psychiatry for LGBTQ Care, Communication, and Advocacy. Acad Psychiatry 2019;43(3):306-10. https://doi.org/10.1007/s40596-019-01024-y

33. Risdon C, Cook D, Willms D. Gay and lesbian physicians in training: a qualitative study. CMAJ 2000;162(3):331-4. PMID: 10693588 